\title{
On functions that solve Mulholland inequality and on compositions of such functions
}

\author{
Milan Petrík ${ }^{1,2}$ \\ ${ }^{1}$ Institute of Computer Science, Academy of Sciences, Prague, Czech Republic. \\ ${ }^{2}$ Faculty of Science, Masaryk University, Brno, Czech Republic.
}

\begin{abstract}
Two results related to Mulholland inequality are presented. First, there are functions that are not geo-convex but solve Mulholland inequality; thus Mulholland's condition is not necessary. Second, the set of functions that solve Mulholland inequality is not closed with respect to compositions. As a corollary, the dominance relation on the set of strict triangular norms is not transitive.

The proofs of both the results are of geometric nature and benefit from the level set plots of the pseudo-additions generated by the functions in question.
\end{abstract}

Keywords: Mulholland inequality, Minkowski inequality, strict triangular norm, dominance relation.

\section{Introduction}

Mulholland inequality is a functional inequality which has been introduced by H. P. Mulholland in his paper [11] from 1950 as a generalization of the Minkowski inequality which represents the triangular inequality for $p$-norms. In the same paper, Mulholland has also provided a sufficient condition for the fulfillment of the inequality. Later, in 1984, Tardiff has shown that Mulholland inequality is closely related to the relation of dominance between strict triangular norms and has provided a different sufficient condition [21]. In 1999 Schweizer posed a question [19] on comparing Mulholland's and Tardiff's condition. This question has been answered in 2002 by Jarczyk and Matkowski who demonstrated [7] that the Tardiff's condition implies Mulholland's condition. An alternative proof has been also given by Baricz [3] in 2010.

The two following questions have however remained open:

Question 1.1 Is Mulholland's condition also necessary?

Question 1.2 Is the set of functions solving Mulholland inequality closed with respect to their compositions?

In this paper we intend to bring the answers. Recall that since Mulholland inequality is closely related to the dominance relation on the set of strict

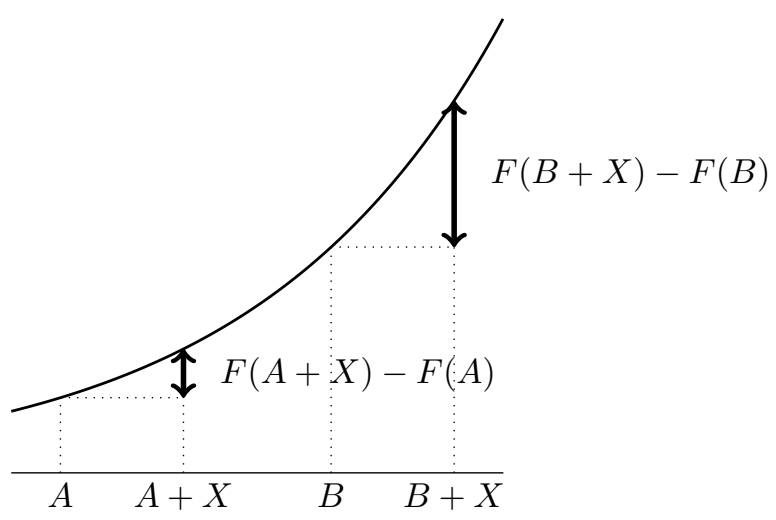

Figure 1: Illustration of the property of an increasing real convex function $F$.

triangular norms. Therefore the answer to the second question gives also an answer to the question whether the dominance relation on the class of strict triangular norms is transitive.

\section{Basic notions}

We denote by $\mathbb{R}_{0}^{+}$the set of positive real numbers with zero and by $\mathbb{R}^{+}$the set of positive real numbers without zero.

Definition 2.1 A function $f: I \rightarrow \mathbb{R}$, defined on a real interval $I \subseteq \mathbb{R}$, is convex resp. geo-convex (or geometrically convex [Y]) if, and only if,

$$
\begin{aligned}
f(\alpha x+(1-\alpha) y) & \leq \alpha f(x)+(1-\alpha) f(y), \\
f\left(x^{\alpha} \cdot y^{1-\alpha}\right) & \leq f^{\alpha}(x) \cdot f^{1-\alpha}(y)
\end{aligned}
$$

holds respectively for every $x, y \in I$ and for every $\alpha \in[0,1]$.

Note that $f$ is geo-convex if, and only if, $F=$ $\log \circ f \circ \exp$ is convex. Note also that if $f$ is convex, or if it is an increasing bijection, then it is, necessarily, continuous.

Definition 2.2 Let $f: \mathbb{R}_{0}^{+} \rightarrow \mathbb{R}_{0}^{+}$be an increasing bijection. The pseudo-addition generated by $f$ is a binary operation

$$
*: \mathbb{R}_{0}^{+} \times \mathbb{R}_{0}^{+} \rightarrow \mathbb{R}_{0}^{+}
$$

given by

$$
x *_{f} y=f^{-1}(f(x)+f(y))
$$



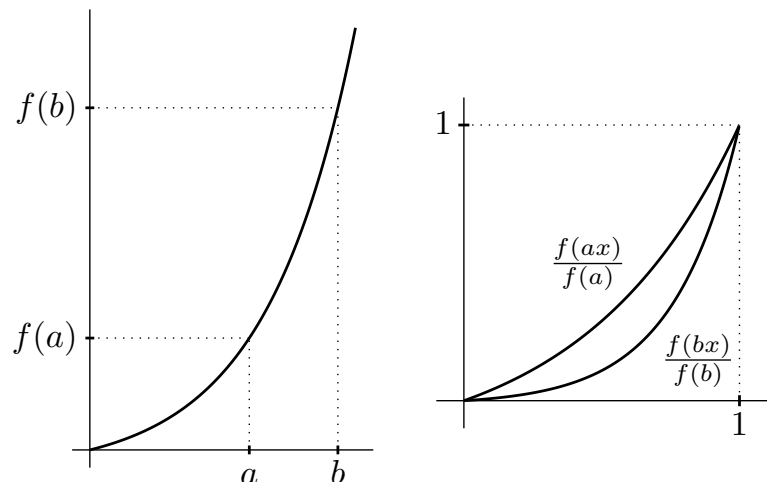

Figure 2: Graph of the bijection $f$ (left) and graphs of the functions $f(a x) / f(a)$ and $f(b x) / f(b)$ compared (right). The latter graphs are identical to the graph of $f$ on $[0, a]$ and $[0, b]$, respectively, scaled to the unit square $[0,1] \times[0,1]$.

for all $x, y \in \mathbb{R}_{0}^{+}$. A level set of $*_{f}$ in the level $a \in \mathbb{R}_{0}^{+}$is the set

$$
L_{a}^{f}=\left\{(x, y) \in \mathbb{R}_{0}^{+} \times \mathbb{R}_{0}^{+} \mid x *_{f} y=a\right\} .
$$

$A$ level cut of $*_{f}$ in the level $a \in \mathbb{R}_{0}^{+}$is the set

$$
\Lambda_{a}^{f}=\left\{(x, y) \in \mathbb{R}_{0}^{+} \times \mathbb{R}_{0}^{+} \mid x *_{f} y \leq a\right\} .
$$

Notice that if $f$ is convex then the level cuts of $*_{f}$ are convex sets.

Let $\mathcal{S}$ be the power-set of $\mathbb{R}_{0}^{+} \times \mathbb{R}_{0}^{+}$. On $\mathcal{S}$, the operation of Minkowski sum, $+: \mathcal{S} \times \mathcal{S} \rightarrow \mathcal{S}$, is defined by

$$
A+B=\{(x+u, y+v) \mid(x, y) \in A,(u, v) \in B\}
$$

for any $A, B \in \mathcal{S}$. A positive real multiple of $A \in \mathcal{S}$ is defined by

$$
\alpha A=\{(\alpha x, \alpha y) \mid(x, y) \in A\}
$$

where $\alpha \in \mathbb{R}_{0}^{+}$. The following facts can be observed for every $A, B, C \in \mathcal{S}$ and for every $\alpha, \beta \in \mathbb{R}_{0}^{+}$:

$$
\begin{aligned}
A+B & =B+A, \\
(A+B)+C & =A+(B+C), \\
(\alpha+\beta) A & =\alpha A+\beta A, \\
A \subseteq B & \Rightarrow A+C \subseteq B+C, \\
A \subseteq B & \Rightarrow \alpha A \subseteq \alpha B .
\end{aligned}
$$

We define a relation $\leq$ on the set of all the level cuts of the pseudo-addition $*_{f}$ by

$$
\Lambda_{a}^{f} \leq \Lambda_{b}^{f} \quad \text { iff } \quad \frac{1}{a} \Lambda_{a}^{f} \subseteq \frac{1}{b} \Lambda_{b}^{f}
$$

This relation is reflexive and transitive, however, it is not anti-symmetric. Thus $\leq$ is a pre-order.

\section{Mulholland inequality and Mulholland's condition}

An increasing bijection $f: \mathbb{R}_{0}^{+} \rightarrow \mathbb{R}_{0}^{+}$is said to solve Mulholland inequality if

$$
\begin{aligned}
f^{-1}( & f(x+u)+f(y+v)) \\
& \leq f^{-1}(f(x)+f(y))+f^{-1}(f(u)+f(v))
\end{aligned}
$$

holds for all $x, y, u, v \in \mathbb{R}_{0}^{+}$. Using the notion of pseudo-addition, we reformulate the formula above to

$\forall x, y, u, v \in \mathbb{R}_{0}^{+}:(x+u) *_{f}(y+v) \leq\left(x *_{f} y\right)+\left(u *_{f} v\right)$.

As you can see, we have obtained the definition of the dominance relation between the operations + and $*_{f}$. Therefore, $f: \mathbb{R}_{0}^{+} \rightarrow \mathbb{R}_{0}^{+}$satisfies Mulholland inequality if, and only if, + dominates the pseudo-addition $*_{f}$ generated by $f$.

Further, using the notion of level cuts, we reformulate the Mulholland inequality formula to

$$
\forall a, b \in \mathbb{R}_{0}^{+}: \quad \Lambda_{a}^{f}+\Lambda_{b}^{f} \subseteq \Lambda_{a+b}^{f} .
$$

The latter inequality allows an intuitive geometric representation which is illustrated in Figure 3.

By $M I$ we denote the set of all the bijections that solve Mulholland inequality. In his paper [11], Mulholland has provided the following sufficient condition:

Theorem 3.1 Let $f: \mathbb{R}_{0}^{+} \rightarrow \mathbb{R}_{0}^{+}$be an increasing bijection. If both $f$ and $\log \circ f \circ \exp$ are convex, i.e., if $f$ is convex and geo-convex, then $f \in M I$.

By $M C$ we denote the set of all increasing bijections on $\mathbb{R}_{0}^{+}$that comply with Mulholland's condition, i.e., that are convex and geo-convex. The result of Mulholland presented in Theorem 3.1 can be stated as $M C \subseteq M I$. The question we are dealing with is whether also $M I \subseteq M C$. The condition of $f$ being convex is necessary [11]. Thus, we need to find the answer whether the condition of $f$ being geo-convex is necessary, as well.

\section{Geo-convexity and $k$-subscalability}

Let $f: \mathbb{R}^{+} \rightarrow \mathbb{R}^{+}$be an increasing bijection and let $F=\log \circ f \circ \exp$; then $F$ is an increasing bijection on $\mathbb{R}$. By the definition, $f$ is geo-convex if, and only if, $F$ is convex. Figure 1 illustrates that $F$ is convex if, and only if,

$$
\begin{aligned}
& \forall A, B, X \in \mathbb{R}, A \leq B: \\
& \quad F(A+X)-F(A) \leq F(B+X)-F(B) .
\end{aligned}
$$

As a corollary, $f$ is geo-convex if, and only if,

$$
\forall a, b, x \in \mathbb{R}^{+}, x \leq 1, a \leq b: \frac{f(a x)}{f(a)} \geq \frac{f(b x)}{f(b)}
$$




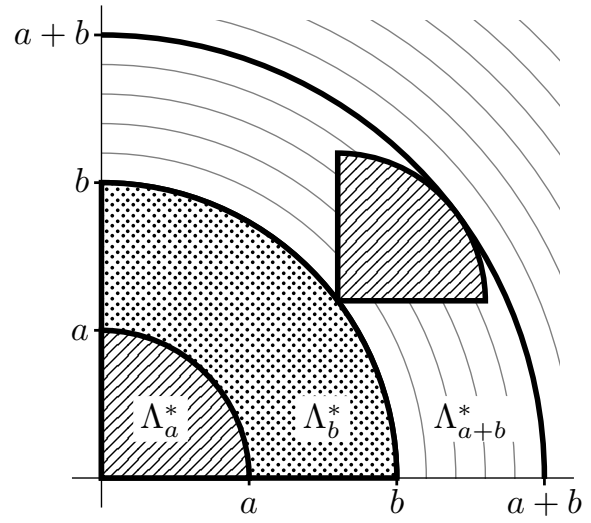

Figure 3: Geometric representation of the Mulholland inequality on the level set plot of the pseudoaddition generated by $f: x \mapsto x^{2}$. Taking a level cut $\Lambda_{a}^{*}$ and shifting it so that its bottom-left corner coincides with the border of $\Lambda_{b}^{*}$, the whole figure must remain within the borders of the level cut $\Lambda_{a+b}^{*}$.

(see an illustration in Figure 2). We weaken this property by introducing a new notion of $k$ subscalability. We say that, for a given $k \in \mathbb{R}_{0}^{+}$, an increasing bijection $f: \mathbb{R}_{0}^{+} \rightarrow \mathbb{R}_{0}^{+}$is $k$-subscalable if

$$
\forall a, b, x \in \mathbb{R}^{+}, x \leq 1, b-a \geq k: \frac{f(a x)}{f(a)} \geq \frac{f(b x)}{f(b)} .
$$

Note that $f$ is geo-convex if, and only if, it is 0subscalable.

We present further the following characterization of convex increasing bijections on $\mathbb{R}_{0}^{+}$that are geoconvex.

Proposition 4.1 [15, Proposition 13] Let $f: \mathbb{R}_{0}^{+} \rightarrow \mathbb{R}_{0}^{+}$be a convex increasing bijection. Then it is geo-convex if, and only if, there exists a sequence $\left(g_{i}: \mathbb{R}_{0}^{+} \rightarrow \mathbb{R}_{0}^{+}\right)_{i \in \mathbb{N}}$ of power functions $g_{i}: x \mapsto q_{i} x^{p_{i}}, p_{i}, q_{i} \in \mathbb{R}_{0}^{+}, p_{i} \geq 1$, such that

$$
f=\bigvee_{i \in \mathbb{N}} g_{i} .
$$

\section{Larger class of functions solving Mulholland inequality}

As it has been proven [12], if

$$
\forall x \in \mathbb{R}^{+}, x \leq 1: \frac{f(a x)}{f(a)} \geq \frac{f(b x)}{f(b)}
$$

for some given $a, b \in \mathbb{R}_{0}^{+}, a \leq b$, then we have

$$
\Lambda_{a}^{f}+\Lambda_{b}^{f} \subseteq \Lambda_{a+b}^{f} .
$$

Therefore, if a convex increasing bijection $f: \mathbb{R}_{0}^{+} \rightarrow$ $\mathbb{R}_{0}^{+}$is $k$-subscalable for some $k \in \mathbb{R}_{0}^{+}$then we have (6) for every $a, b \in \mathbb{R}_{0}^{+}$such that both $a$ and $b$ are greater than or equal to $k$. Thus, we want to

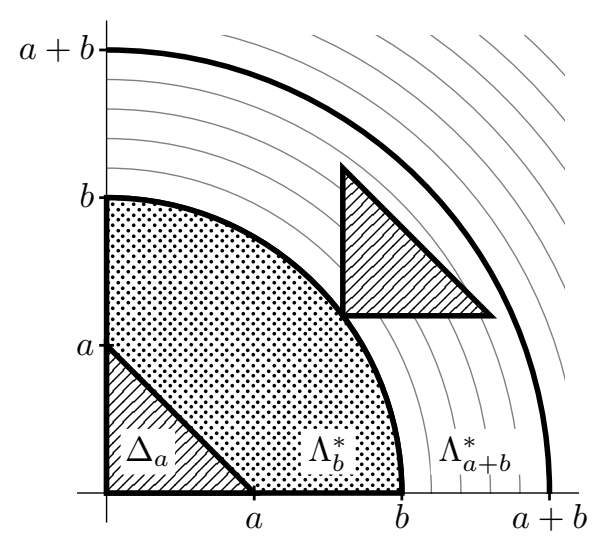

Figure 4: If $\Lambda_{a}^{*}=\Delta_{a}$ then $\Lambda_{a}^{*}+\Lambda_{b}^{*} \subseteq \Lambda_{a+b}^{*}$ always holds for pseudo-additions generated by convex increasing bijections.

find a sufficient condition such that (6) holds also if $a<k$ or $b<k$. We present here a rather simple one; we believe, however, that this condition is not necessary and that the problem deserves a further investigation.

Proposition 5.1 [12] Let $f: \mathbb{R}_{0}^{+} \rightarrow \mathbb{R}_{0}^{+}$be a convex increasing bijection that is linear on the interval $[0, k]$ for some $k \in \mathbb{R}_{0}^{+}$, i.e., if there exists $r \in \mathbb{R}^{+}$ such that $f(x)=r x$ for all $x \in[0, k]$. Then we have $\Lambda_{a}^{f}+\Lambda_{b}^{f} \subseteq \Lambda_{a+b}^{f}$ for every $a, b \in \mathbb{R}_{0}^{+}$such that $a \leq k$ or $b \leq k$.

This idea of the proposition is illustrated in Figure 4 . As a corollary, we obtain the following theorem:

Theorem 5.2 [12] Let $f: \mathbb{R}_{0}^{+} \rightarrow \mathbb{R}_{0}^{+}$be a convex increasing bijection which, for some $k \in \mathbb{R}_{0}^{+}$, is $k$ subscalable and linear on the interval $[0, k]$. Then $f$ solves Mulholland inequality, i.e., $f \in M I$.

For a given $k \in \mathbb{R}_{0}^{+}$, we denote by $S_{k}$ the set of all $k$-subscalable bijections and by $L_{k}$ the set of all bijections that are linear on $[0, k]$. Further, we introduce the set $L S_{k}=L_{k} \cap S_{k}$ and the set

$$
L S=\bigcup_{k \in \mathbb{R}_{0}^{+}} L S_{k} .
$$

Thus, the set $L S$ represents all the functions that accord with the assumptions of Theorem 5.2. According to the results described here, we have $M C \subseteq L S \subseteq M I$ since $L S_{0}=M C$.

Example 5.3 The increasing bijection $f: \mathbb{R}_{0}^{+} \rightarrow$ $\mathbb{R}_{0}^{+}$is defined, for all $x \in \mathbb{R}_{0}^{+}$, as

$$
g(x)= \begin{cases}\frac{5}{3} x & \text { if } x \in[0,1], \\ \frac{7}{3} x-\frac{2}{3} & \text { if } x \in] 1,2], \\ x^{2} & \text { if } x \in] 2, \infty[.\end{cases}
$$

The graph of the function $g$ is illustrated in Figure 5 and the generated pseudo-addition $*_{g}$ is illustrated in Figure 6. 


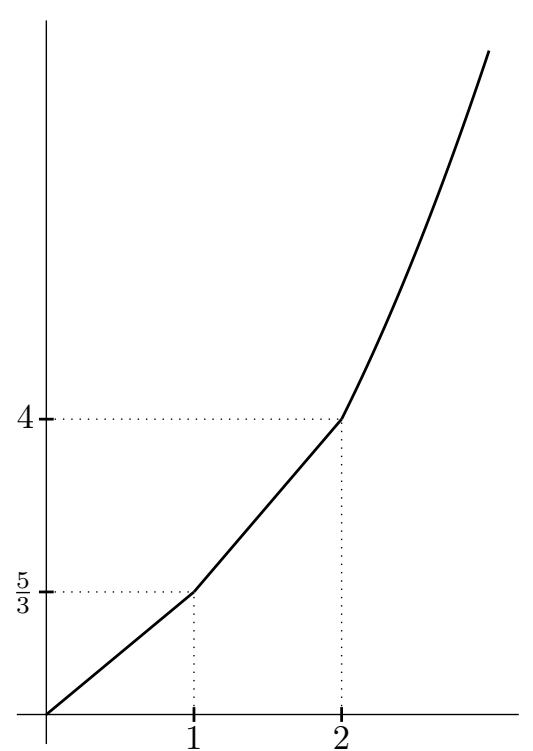

Figure 5: Left: Graph of the bijection $g$ given by Example 5.3 which is 1-subscalable and 1-linear but not geo-convex.

Proposition 4.1 implies that $g \notin M C$. Moreover, the previous result [12] has shown that $g \in M I$. Therefore Example 5.3 shows that $M C \subsetneq L S$.

Notice that the question whether $L S \subsetneq M I$ or $L S=M I$ remains still open although we conjecture that the first case is the true.

\section{Mulholland inequality and dominance of strict triangular norms}

Definition 6.1 $A$ triangular norm (or a t-norm for short) [2, 9] is a commutative, associative, and non-decreasing binary operation $*:[0,1] \times[0,1] \rightarrow$ $[0,1]$ with neutral element 1. A t-norm is said to be strict if there exists a decreasing bijection $\varphi:[0,1] \rightarrow[0, \infty]$ such that

$$
x * y=\varphi^{-1}(\varphi(x)+\varphi(y))
$$

for all $x, y \in[0,1]$; the bijection $\varphi$ is then called the generator of $*$.

T-norms are studied nowadays mainly in the framework of the basic logic $[5,6]$ and the monoidal t-norm based logic [4] which are both prototypical fuzzy logics; particularly, the real unit interval $[0,1]$ endowed with a strict t-norm is isomorphic to the standard semantics of the product logic which is a special case of the basic logic. Nevertheless, originally the t-norms have been introduced within the framework of probabilistic metric spaces $[18,10]$ where they establish the triangular inequality of the probabilistic metrics.

Dominance is a binary relation on a set of $n$-ary operations. Particularly, a t-norm $*$ dominates a

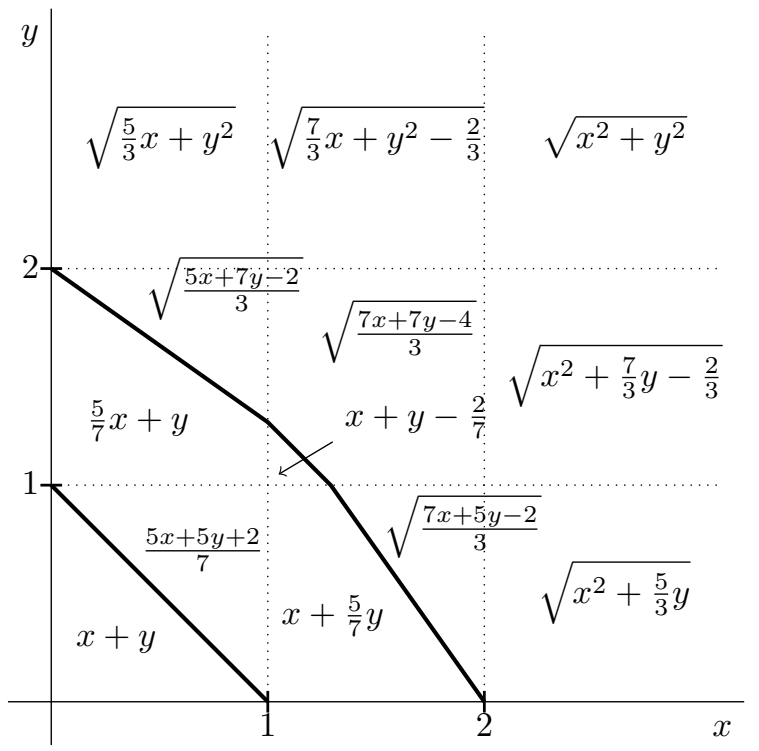

Figure 6: Scheme of the functional values of the pseudo-addition generated by the bijection $g$ given by Example 5.3.

t-norm $\diamond$, denoted by $* \gg \diamond$, if

$\forall x, y, u, v \in[0,1]: \quad(x \diamond y) *(u \diamond v) \geq(x * u) \diamond(y * v)$.

The motivation to study dominance of t-norms comes from Tardiff [22] who recognized that dominance plays an important role when constructing Cartesian products of probabilistic metric spaces. Tardiff has also shown that the dominance of strict t-norms is closely related to Mulholland inequality [21, Theorem 3].

Proposition 6.2 Let $*_{1}:[0,1] \times[0,1] \rightarrow[0,1]$ and $*_{2}:[0,1] \times[0,1] \rightarrow[0,1]$ be two strict $t$-norms given by the generators $\varphi_{1}:[0,1] \rightarrow[0, \infty]$ and $\varphi_{1}:[0,1] \rightarrow[0, \infty]$, respectively, i.e.,

$$
\begin{aligned}
& x *_{1} y=\varphi_{1}^{-1}\left(\varphi_{1}(x)+\varphi_{1}(y)\right), \\
& x *_{2} y=\varphi_{2}^{-1}\left(\varphi_{2}(x)+\varphi_{2}(y)\right)
\end{aligned}
$$

for $x, y \in[0,1]$. Then $*_{2}$ dominates $*_{1}$, i.e., $*_{1} \gg$ $*_{2}$, if, and only if, $f=\varphi_{2} \circ \varphi_{1}^{-1}$ solves Mulholland inequality.

Let us remark that, recently, this correspondence has been enlarged to all continuous Archimedean triangular norms [13].

It can be checked easily that the dominance relation is reflexive and anti-symmetric. Nevertheless, it remained an open question for a long time whether it is also transitive, and thus an order relation [1, Problem 17]. This question has been answered recently by Sarkoci [16] who has given a negative answer for general continuous t-norms. However, for the class of strict t-norms the question remained open. Nevertheless, it has been revealed $[8,14,17,20]$ that for many significant subclasses of strict t-norms the dominance relation is transitive. Proposition 6.2 implies the following. 


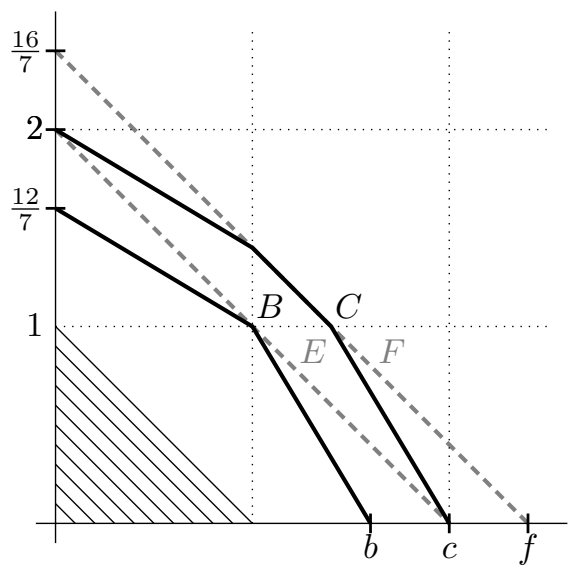

Figure 7: Illustration of the level set plot of the pseudo-addition $*_{g}$ generated by the function $g$ from Example 7.1 with denoted level sets in question.

Proposition 6.3 The dominance relation on the set of strict t-norms is transitive if the set $M I$ is closed with respect to compositions; this means that if $f_{1} \in M I$ and $f_{2} \in M I$ then also $f_{1} \circ f_{2} \in M I$.

\section{The set $L S$ is not closed with respect to compositions}

Example 7.1 Let $g$ be the function from Example 5.3 and let $h: \mathbb{R}_{0}^{+} \rightarrow \mathbb{R}_{0}^{+}: x \mapsto x^{2}$. The composition of these two functions, $f=g \circ h$, does not solve Mulholland inequality, i.e., $f \notin M I$.

This statement can be proven simply by evaluating the inequality (1), e.g., for $x=y=1$ and for $u=v<0.074$. Nevertheless, we present here also a geometric proof illustrated in Figure 7 and Figure 8 . By + we denote the common real addition of non-negative real numbers.

Consider the pseudo-additions $*_{g}$ and $*_{f}$ generated by $g$ and $f$, respectively.

Figure 7 illustrates the level set plot of $*_{g}$ with emphasized level sets in $b=\frac{12}{7}$ and $c=2$; denote them by $B$ and $C$, respectively. There are, furthermore, two line segments parallel to the level sets of + ; one passes through the point $(1,1)$, denote it by $E$, and the second through the middle segment of $C$, denote it by $F$. The edge vertices of $E$ are $(0, c)$ and $(c, 0)$; the edge vertices of $F$ are $(0, f)$ and $(f, 0)$ where $f=\frac{16}{7}$. Stress out that $E$ and $F$ are not level sets of $* g$.

Figure 8 illustrates the level set plot of $*_{f}$. Note that it can be seen as the image of the level set plot of $g$ when applying the mapping $m: \mathbb{R}_{0}^{+} \times \mathbb{R}_{0}^{+} \rightarrow \mathbb{R}_{0}^{+}$ defined by

$$
m(x, y)=(\sqrt{x}, \sqrt{y})
$$

Observe that all the level sets in $[0,1]$, as well as the images of $E$ and $F$, are quarter-circles centered at

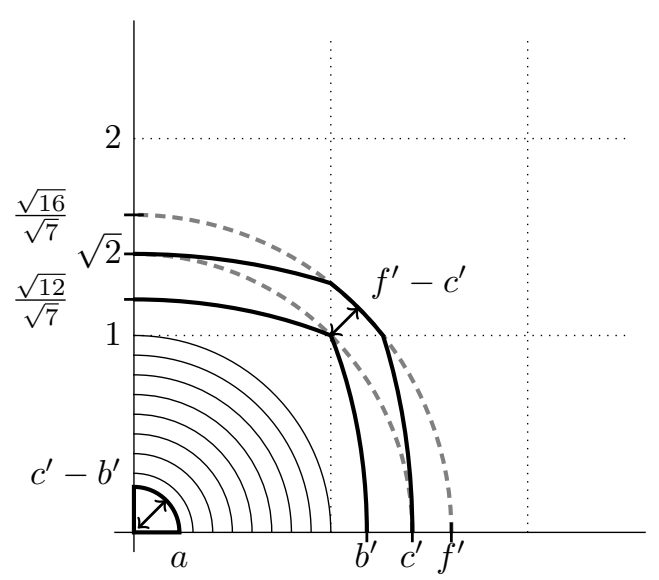

Figure 8: Illustration of the level set plot of the pseudo-addition $*_{f}$ generated by the function $f$ from Example 7.1 with denoted level sets in question. It can be seen that the distance $f^{\prime}-c^{\prime}$ is shorter than the distance $a^{\prime}=c^{\prime}-b^{\prime}$.

$(0,0)$. Denote

$$
\begin{aligned}
b^{\prime} & =\sqrt{b}, \\
c^{\prime} & =\sqrt{c}, \\
a^{\prime} & =c^{\prime}-b^{\prime}, \\
f^{\prime} & =\sqrt{f} .
\end{aligned}
$$

The point of the proof is to show that

$$
\Lambda_{a^{\prime}}^{f}+\Lambda_{b^{\prime}}^{f} \nsubseteq \Lambda_{c^{\prime}}^{f}
$$

This is indeed true since the distance $l=f^{\prime}-c^{\prime}$ denoted in Figure 8 is strictly shorter than $a^{\prime}$ which follows from the fact that $x \mapsto \sqrt{x}$ is a concave increasing bijection on $\mathbb{R}_{0}^{+}$and thus from

$$
c-b=f-c
$$

follows

$$
\sqrt{c}-\sqrt{b}<\sqrt{f}-\sqrt{c} .
$$

According to Example 5.3 we have $g \in L S$ and according to Proposition 4.1 we have $h \in M C$. Thus $g$ and $h$ give us an example of two functions which solve Mulholland inequality but their composition does not.

\section{Concluding remarks}

A new condition which is sufficient in order to give a solution for Mulholland inequality has been introduced. Moreover, this new condition delimits strictly more solutions than the one of Mulholland. However, it still remains an open question whether this new condition describes all the possible solutions of Mulholland inequality. We conjecture that this is not the case.

Further, it has been shown that the set $L S$, as well as the set $M I$, is not closed with respect to 
compositions. As it can be easily verified, the set $M C$ is closed. Thus there is an interesting open question what is the maximal subset of $M I$ that is closed with respect to compositions.

\section{Acknowledgments}

This work was partially supported by the Czech Science Foundation under Project P201/12/P055 and partially by ESF Project CZ.1.07/2.3.00/20.0051 Algebraic methods in Quantum Logic of the Masaryk University. Supported by

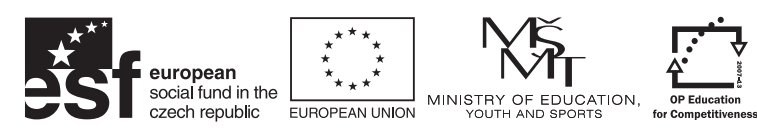
INVESTMENTS IN EDUCATION DEVELOPMENT

\section{References}

[1] C. Alsina, M. J. Frank, and B. Schweizer. Problems on associative functions. Aequationes Mathematicae, 66(1-2):128-140, 2003.

[2] C. Alsina, M. J. Frank, and B. Schweizer. Associative Functions: Triangular Norms and Copulas. World Scientific, Singapore, 2006.

[3] Á. Baricz. Geometrically concave univariate distributions. Journal of Mathematical Analysis and Applications, 363(1):182-196, 2010. DOI: $10.1016 /$ j.jmaa.2009.08.029.

[4] F. Esteva and L. Godo. Monoidal t-norm based logic: towards a logic for left-continuous tnorms. Fuzzy Sets and Systems, 124:271-288, 2001.

[5] P. Hájek. Basic fuzzy logic and BL-algebras. Soft Computing, 2:124-128, 1998.

[6] P. Hájek. Metamathematics of Fuzzy Logic. Kluwer, Dordrecht, 1998.

[7] W. Jarczyk, J. Matkowski. On Mulholland's inequality. Proc. Amer. Math. Soc., 130(11):3243-3247, 2002.

[8] M. Kauers, V. Pillwein, and S. Saminger-Platz. Dominance in the family of Sugeno-Weber tnorms Fuzzy Sets and Systems, 181(1):74-87, 2011. DOI: $10.1016 /$ j.fss.2011.04.007

[9] E. P. Klement, R. Mesiar, and E. Pap. Triangular Norms, vol. 8 of Trends in Logic. Kluwer Academic Publishers, Dordrecht, Netherlands, 2000 .

[10] K. Menger. Statistical metrics. Proc. Nat. Acad. Sci. U.S.A., 8:535-537, 1942.

[11] H. P. Mulholland. On generalizations of Minkowski's inequality in the form of a triangle inequality. Proc. London Math. Soc., 51(2):294-307, 1950.

[12] M. Petrík, M. Navara, and P. Sarkoci. Alternative proof of Mulholland's theorem and new solutions to Mulholland inequality. ISMVL 2013: IEEE 43rd International Symposium on Multiple-Valued Logic, Toyama, Japan, May 21-24, 2013.
[13] S. Saminger-Platz, B. De Baets, and H. De Meyer. A generalization of the Mulholland inequality for continuous Archimedean t-norms. Journal of Mathematical Analysis and Applications, 345:607-614, 2008.

[14] S. Saminger-Platz. The dominance relation in some families of continuous archimedean t-norms and copulas. Fuzzy Sets and Systems, 160:2017-2031, 2009. DOI: 10.1016/j.fss.2008.12.009

[15] P. Sarkoci. Dominance Relation for Conjunctors in Fuzzy Logic. Ph.D. thesis, January 2007.

[16] P. Sarkoci. Dominance is not transitive on continuous triangular norms. Aequationes Math., 75:201-207, 2008.

[17] P. Sarkoci. Domination in the families of Frank and Hamacher t-norms. Kybernetika, 41:345356, 2005.

[18] B. Schweizer, A. Sklar. Probabilistic Metric Spaces. Dover Publications, Mineola, NewYork, 2005.

[19] A. Sklar. Remark and Problem, Report of meeting, 37th International Symposium on Functional Equations (Huntington, 1999). Aequationes Mathematicae, 60:187-188, 2000.

[20] H. Sherwood. Characterizing dominates on a family of triangular norms. Aequationes Mathematicae, 27:255-273, 1984.

[21] R. M. Tardiff. On a generalized Minkowski inequality and its relation to dominates for tnorms. Aequationes Mathematicae, 27(3):308316, 1984.

[22] R. M. Tardiff. Topologies for probabilistic metric spaces. Pacific Journal of Mathematics, 65(1):233-251, 1984. 